\title{
Sheet-Metal Shop Scheduling Considering Makespan and Flow Time Criteria
}

\author{
H. Crauwels ${ }^{*}, 1$, B. Verlinden ${ }^{2}$, D. Cattrysse ${ }^{3}$ and D. Van Oudheusden ${ }^{3}$ \\ ${ }^{1}$ Hogeschool voor Wetenschap \& Kunst, campus De Nayer, J. De Nayerlaan 5, B-2860 Sint-Katelijne-Waver, Belgium \\ ${ }^{2}$ Sirris, Celestijnenlaan 300C, B-3001 Leuven, Belgium \\ ${ }^{3}$ K.U. Leuven, Centre for Industrial Management, Celestijnenlaan 300A, B-3001 Leuven, Belgium
}

\begin{abstract}
Sheet-metal parts typically follow a unidirectional flow in the sheet-metal shop. In the first cutting stage, a large sheet is cut to different unfolded parts with a laser cutting machine. To avoid waste material different parts are combined on a sheet. Next, the 2D parts are transformed to 3D products with air bending. In this bending stage, timeconsuming set-ups between production layouts are reduced as much as possible. Separate optimisation of cutting and air bending causes the optimisation benefits to counteract one another. Integrated models have been proposed for both singleand multiple-machine classes, but calculation times are too high and avoidable changeovers still occur.

In this paper, by applying variable neighbourhood search with a number of different starting solutions, local optima of good quality are determined for minimising the makespan and the total flow time for both the single-machine and the multi-machine classes. Because the two performance measures are important for a good production plan, bicriteria optimisation by means of a simultaneous and a hierarchical approach, is also considered. Compared to the mathematical programming models for the combined cutting and bending operations, both quality and required computation time are improved for several real-life instances.
\end{abstract}

Keywords: Sheet-metal shop, single-machine planning, multiple-machine planning, bicriteria scheduling, variable neighbourhood search.

\section{INTRODUCTION}

Due to the introduction of new processes such as laser cutting and incremental forming, sheet-metalworking has been reconsidered as a viable option for both structural components and designer products. A typical sheet-metal part follows a number of operations as can be seen from Fig. (1). In a small company, just one laser cutting machine and one press brake are available. Larger companies can have multiple press brakes and/or laser machines.

First a flat blank is cut with the laser machine to obtain the unfolded workpiece. Since material is an important cost factor, different parts requiring the same material and thickness are combined on a larger sheet to reduce the waste material as much as possible (the so-called nesting problem). After the unfolded parts have been cut, they are sent to the press brake for bending. By applying a vertical force, the unfolded workpiece is forced into the die and a $3 \mathrm{D}$ product is produced. The geometrical properties of the $3 \mathrm{D}$ product determine the type of punch and die, based on collision avoidance between part, tools and machine. A single part consisting of multiple bend lines might demand different tool sets, necessitating a specific production layout. Interchanging those layouts is time consuming due to manual actions and should be avoided as much as possible.

*Address correspondence to this author at the Hogeschool voor Wetenschap \& Kunst, campus De Nayer, J. De Nayerlaan 5, B-2860 Sint-KatelijneWaver, Belgium; E-mail: herman.crauwels@denayer.wenk.be
In many situations, cutting and air bending can be considered as two operations in a flow shop where no large inventory is allowed between the two machines. Preferably, production planning for the laser machine and the press brake should be integrated to avoid counteracting benefits when optimising separately. Several optimisation models can be found in Verlinden et al. [1,2]. These models minimise the number of set-ups at the press brake and the makespan of the parts by reformulating the problem as a well-known vehicle routing problem (Toth and Vigo [3], Golden et al. [4]). Different parts are combined on a sheet and truck capacity constraints induce the minimisation of waste material. Sequence-dependent changeovers between production layouts are used. Good results can be generated, but calculation times are within the range of dozens of minutes, while the production plan should be generated just before starting production, in a very short time span. In addition, individual sheets following one another still lead to avoidable set-ups.

Depending on the work volume and the thickness of the metal sheets, multiple press brakes and/or multiple laser machines can be used. An optimisation model for the integrated production planning of the multiple laser machines and press brakes is developed by Verlinden et al. [2]. The objective is to minimise the makespan. To reduce the complexity of the model, first the single laser machine/single press brake problem (based on the vehicle routing problem) is solved. The resulting metal sheets 


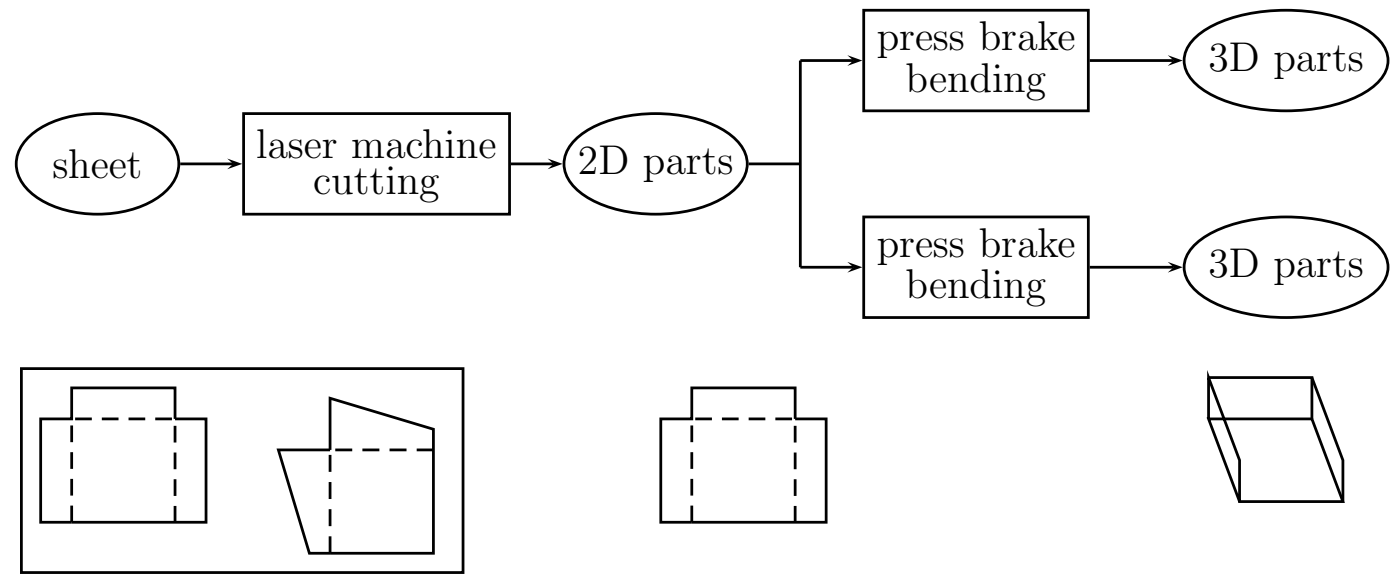

Fig. (1). Sheet-metal operations.

(composed of several workpieces) are then the input for the multiple-machine models. A side effect of this two-stage approach is that all workpieces from a single sheet are cut by the same laser machine and then moved to a single press brake for bending. For the material handling operations, this is a nice feature.

Formally, three different classes of problems can be distinguished: single laser machine/multiple related press brakes (SLRP), single laser machine/multiple unrelated press brakes (SLUP) and multiple unrelated laser machines/multiple unrelated press brakes (ULUP). For the related machine class, cutting and bending times are independent of the assigned laser cutting machine and press brake, respectively. For the unrelated classes, $50 \%$ of the machines are considered fast machines; and the slower machines require $20 \%$ more time for processing a sheet (either cutting or bending).

For the developed mathematical programming models, the required computation time for solving these models exactly is too large. Therefore, a variable neighbourhood search (VNS) is proposed by Verlinden et al. [2] to tackle these problems. The basic idea of VNS is the change of neighbourhoods in the search for a better solution (Mladenović and Hansen [5], Hansen and Mladenović [6]). VNS starts by applying a descent method to a local minimum and then proceeds by using different neighbourhood structures. In the method, the current solution is superseeded by a new one when a better solution has been found.

In this paper, the VNS procedures are extended in a number of ways. Firstly, two different objective functions are considered for both the single-machine and the multiplemachine classes: minimising the makespan and minimising the total flow time on the press brake(s). Because the ready time of all jobs is assumed to be zero, this second measure is equal to the sum of completion times on the press brake(s). It is obvious that makespan is an important criterion: a company wants to complete a set of workpieces as quickly as possible. A short total production time can help to reduce the delivery time, resulting in a competitive advantage. But also the minimised total flow time can be of interest for a company. Total flow time is proportional to work-in-process.
As already indicated, no large inventory is allowed between the cutting and air bending machines. Less inventory in the sheet-metal shop reduces the total cost of the final product which can also be advantageous. The differences between the two resulting production plans are clearly indicated. Secondly, for the multiple-machine classes an integrated approach is suggested. Instead of using the composed sheets from the single-machine solution as the input for the multiple-machine problem, the procedure constructs a production plan starting from the individual workpieces. The results are compared with the production plan of the twostage approach.

Because schedules made for makespan minimisation and schedules made for total flow time minimisation can differ a lot, a bicriteria solution should be preferred when the constructed schedule has to perform well on both criteria. A survey on bicriteria scheduling can be found in T'kindt and Billaut [7]. Solution approaches for standard problems related to the problem considered in this article have already been suggested. Gupta and Ho [8] consider the parallel machines problem for finding an optimal schedule with the smallest total flow time among the schedules with optimal makespan. Bagga and Bhambani [9] propose a simple procedure for obtaining the sequence that minimises total flow time subject to minimum makespan in the flowshop problem. Allahverdi [10] compares a number of heuristic methods for the flowshop problem with bicriteria of makespan and mean flow time. Hendizadeh et al. [11] also consider the flowshop problem but they also take into account sequence-dependent set-up times. However, to the best of our knowledge, no solution procedure has been developed for the problem considered in this paper in the context of bicriteria optimisation.

Another point of issue is the required number of sheets for the given set of workpieces. In the mathematical modeling approach of Verlinden et al. [2] this number is determined in advance by dedicated nesting software. In a practical setting, it is not guaranteed that the VNS procedure of Verlinden et al. [2] can construct a production plan with that small number of sheets. Therefore, the VNS is adapted in such a way that a solution with the actual minimal number of sheets becomes more likely. 
In Section 2, a formal statement of the problem is given. Section 3 and 4 introduce the heuristic solution method for the single-machine class and the multiple-machine classes, respectively. The problem of bicriteria optimisation is considered in Section 5. Section 6 reports on computational experiments. Some concluding remarks are offered in Section 7.

\section{PROBLEM FORMULATION}

To state the paper's problem precisely, $T$ jobs are considered. Each job corresponds to the fabrication of a number of identical 3D parts, or workpieces. The characteristics of a job $j$ are the number of workpieces $n_{j}$, the kind of material (e.g. steel), the thickness of the material and the required surface of the 2D part that has to be cut out of a sheet of metal. Also given is the laser cutting time $c_{j}$ and the press brake bending time $p_{j}$. For each job a specific production layout $f$ is specified on the press brake. In total, there are $n=\sum n_{j}$ workpieces. At the laser machine a set-up is required before the sheet can be cut. This time is composed of three elements: a constant time for the basic set-up (positioning the sheet and loading the cutting programme), a time period depending on the thickness of the sheet, and, when there is a change of material, an extra time for changing the lenses and nozzles of the laser machines. A sequence-dependent set-up time $s_{f g}$ is incurred at the press brake whenever a job requiring production layout $g$ is processed immediately after a job requiring a different production layout $f$. Also, an initial set-up time $s_{0 f}$ is required if a job with production layout $f$ is the first to be processed. Each sheet of metal has a surface of which only a fraction $\alpha$ can be used because of the sometimes quite irregular patterns of the 2D parts. The objective is to group together the workpieces on a number of sheets resulting in a production plan that minimises the makespan for the pool of jobs or the total flow time on the press brake(s). The total flow time $\left(\sum F_{i}\right)$ is equal to the sum of all flow times, where the flow time of a sheet corresponds to the completion time $C_{i}$ of the last workpiece of that sheet on the press brake, because the ready time of all required sheets is assumed to be zero. The maximum value of these completion times $C_{i}$ is the makespan $C_{\max }$.

The problem data of an example with $T=7$ jobs is given in Table 1a (job number, number of workpieces, start number of workpiece, cutting and bending time, kind of material, thickness, required area and production layout) and Table 1b (sequence-dependent production layout set-ups on the press brake). The size of a sheet is $3.5 \times 2.5$ metres and $\alpha=0.70$. A production plan with makespan $C_{\max }=82$ and total flow time $\sum F_{j}=266$ is presented in Fig. (2).

Table 1a. Job Characteristics

\begin{tabular}{|c|c|c|c|c|c|c|c|c|}
\hline & $\boldsymbol{n}_{\boldsymbol{j}}$ & & $\boldsymbol{c}_{\boldsymbol{j}}$ & $\boldsymbol{p}_{\boldsymbol{j}}$ & & $\boldsymbol{d}$ & Area & $\boldsymbol{f}$ \\
\hline \hline $\mathbf{1}$ & 2 & 1 & 1 & 1 & $\mathrm{~S}$ & 1.0 & 0.8 & $\mathrm{~L} 4$ \\
\hline $\mathbf{2}$ & 3 & 3 & 2 & 4 & $\mathrm{~S}$ & 1.0 & 1.1 & $\mathrm{~L} 3$ \\
\hline $\mathbf{3}$ & 2 & 6 & 3 & 3 & $\mathrm{~S}$ & 1.0 & 1.5 & $\mathrm{~L} 5$ \\
\hline $\mathbf{4}$ & 1 & 8 & 3 & 3 & $\mathrm{~S}$ & 2.0 & 1.0 & $\mathrm{~L} 2$ \\
\hline $\mathbf{5}$ & 2 & 9 & 4 & 4 & $\mathrm{~S}$ & 2.0 & 0.5 & $\mathrm{~L} 1$ \\
\hline $\mathbf{6}$ & 4 & 11 & 3 & 3 & $\mathrm{SS}$ & 2.0 & 1.3 & $\mathrm{~L} 3$ \\
\hline $\mathbf{7}$ & 6 & 15 & 3 & 2 & $\mathrm{SS}$ & 2.0 & 0.7 & $\mathrm{~L} 4$ \\
\hline
\end{tabular}

Table 1b. Set-Ups

\begin{tabular}{|c|c|c|c|c|c|}
\hline & L1 & L2 & L3 & L4 & L5 \\
\cline { 2 - 6 } & 2 & 4 & 4 & 3 & 6 \\
\hline $\mathbf{L 1}$ & 0 & 2 & 4 & 3 & 4 \\
\hline $\mathbf{L 2}$ & 3 & 0 & 2 & 4 & 3 \\
\hline $\mathbf{L 3}$ & 4 & 2 & 0 & 2 & 2 \\
\hline $\mathbf{L 4}$ & 2 & 3 & 1 & 0 & 3 \\
\hline $\mathbf{L 5}$ & 5 & 5 & 2 & 2 & 0 \\
\hline
\end{tabular}

As indicated in the previous section, several mathematical programming models have been developed by Verlinden et al. [1, 2]. For convenience, the multiplemachine model for minimising makespan is presented in the appendix.

Besides completion time related performance criteria, good schedules can also be characterised by due date related measures, e.g. total and maximum tardiness. For the considered classes of problems, these measures are less important because of the short time horizon, in most cases just one half or a full working day. Another reason is that there can be a number of additional operations (e.g. painting and packaging) that largely determine the delivery time of the final products to the customers.

\section{THE SINGLE-MACHINE CLASS}

Neighbourhood search is a heuristic method that is quite simple and often capable of finding good, but not necessarily optimal solutions very quickly without requiring a huge amount of computational effort. It starts with a known feasible solution and tries to improve upon this solution by making well-defined adjustments. A solution that is

\begin{tabular}{|c|c|c|c|c|c|c|}
\hline $1-2,3-5$ & $6-7$ & $8-10$ & $11-14,15-16$ & $17-20$ & & laser machine \\
\hline$\sqrt{ }$ & $1-2,3-5$ & $6-7$ & $8-10$ & $11-14,15-16$ & $17-20$ & press bral \\
\hline
\end{tabular}

Fig. (2). Production plan. 
generated by such a change to a solution $x$ is called a neighbour of $x$. The neighbourhood $N(x)$, a subset of the solution space, is the set of all neighbours of $x$. During the iterative process, one 'moves' through the solution space from neighbour to neighbour. The move is evaluated by comparing the objective function value of the current solution to that of its neighbour. If the former is worse, the neighbour represents an improving move; if the latter is worse, it is a deteriorating move; if both are the same, it is a neutral move. In the pure descent method, only improving moves are allowed. When no further improvement can be found, the procedure stops resulting in a local optimum, not necessarily the global optimum.

In the past decades several metaheuristics have been developed for escaping from a local optimum by accepting neutral and deteriorating moves in some controlled way. Simulated annealing and tabu search are the most widely spread algorithms (Rayward-Smith et al. [12]). Recently, good quality solutions have been constucted for several combinatorial problems by still another metaheuristic, variable neighbourhood search (Hansen et al. [13]). In this paper, the VNS, developed by Verlinden et al. [2] for the sheet-metal shop is further extended.

A number of issues have to be dealt with when a descent procedure is implemented. The most important is the definition of a move in the neighbourhood. Each type of move determines a different neighbourhood. Five different neighbourhood structures are used in the developed VNS procedure. The move definition is related to the representation of a solution. For the production planning problem, the 'natural' representation is a permutation of the integers $1, \ldots, n$ with $n$ the number of workpieces. Considering this representation, two basic moves can be defined. With shift_workpiece a workpiece is removed from one position in the sequence and inserted at another position (either before or after the original position). Swap_workpiece swaps two workpieces which are not required to be adjacent.

Because problem instances can contain a number of similar workpieces (grouped in a single job), two additional neighbourhood structures are defined. Swap_job swaps two jobs which are not required to be adjacent. This swap is only considered when the two jobs are each on a single sheet. Shift_job removes a job from one position in the sequence and inserts it at another position (either before or after the original position). Again, all the workpieces of a job have to be on the same sheet.

During the search through a neighbourhood, feasibility is maintained. This means that, when different sheets are involved, these four moves are only considered when the two sheets have the same thickness and are of the same material. Also, the total used surface of both sheets has to be smaller than the usable surface of the sheet.

When a sheet contains just one workpiece or only workpieces of the same job, a move of the shift_workpiece or of the shift_job neighbourhood results in the reduction of the number of sheets. Because a production plan with a minimal number of sheets is very important, such a move is carried out whenever the resulting number of sheets is smaller than the number of sheets of the best solution found so far. It is possible that the makespan/total flow time increases by this move, but waste material minimisation is essential in a sheet-metal shop.

For the order in which the neighbourhood is searched, we use a fixed natural lexicographic ordering, i.e. $(1,2),(1,3), \ldots,(1, n),(2,1),(2,3), \ldots,(i, j), \ldots,(n-1, n)$, with $i$ and $j$ the two workpiece or job numbers (for swap moves only pairs where $i<j$ are considered). The first improvement technique is used: each time an improving move is evaluated, it is carried out; and the next iteration continues with the same $i$ and the next $j$ value.

Because several workpieces are grouped together on a sheet, a fifth neighbourhood (insert_sheet) can be defined. The move is carried out by removing a sheet from one position in the sequence and inserting it after some other sheet. For this neighbourhood, the best position in which the sheet can be inserted is considered, and this move is carried out when it results in a better objective function value. Note that it is possible that the production layout required by the last workpiece on a sheet can be the same as the one required by the first workpiece on the next sheet. In that case, it is probably not a good idea to shift that sheet to another position in the sequence. Therefore, in this neighbourhood not only single sheets are considered for shifting but also subsequences of sheets, where the last workpiece of the previous sheet and the first workpiece of the next sheet require the same production layout.

In the VNS procedure a combination of the five neighbourhoods is used. The method starts with the descent method with the swap_workpiece and the shift_workpiece neighbourhood. On the resulting sequence, the descent method based on the the insert_sheet neighbourhood is applied. Finally, the shift_job and the swap_job neighbourhoods are searched. When during one of these five descent procedures an improvement is found, the complete cycle with the five neighbourhoods is repeated until no improvement can be found with one of the five neighbourhoods. In this way, a local optimum relative to five different neighbourhood structures is calculated. Notice that several sequences for searching the different types of neighbourhood have been investigated. The best sequence was selected. For the makespan objective, not only improving moves are accepted but also neutral moves if the total set-up time at the press brake becomes smaller.

The search has to be initialised with a first solution. This solution can be constructed by some heuristic rule or it can be chosen at random. In the proposed approach, six different initial solutions are constructed. For the first four variants, an initial solution is constructed by grouping together workpieces that require the same material and are of the same thickness. A first workpiece is selected and then, among the following workpieces, additional workpieces are searched that have the same material and thickness requirements. These workpieces are added to the same sheet of metal until there is not enough surface anymore on the 
sheet. Then, a new sheet is started. A new sheet is also taken when there are no more workpieces requiring the same material and same thickness. The ordering in which the workpieces are searched, is one of the determinant factors for the quality of the final solution. Therefore, four different orderings of the workpieces are used:

- as given in the problem data input (ordered by thickness);

- the reverse order of the problem data input;

- $\quad$ based on the production layout number: from small to large;

- $\quad$ calculated by the nearest neighbour heuristic: the first workpiece is the one with the smallest average set-up time; following workpieces are added by looking for the smallest set-up time from the actual workpiece to the following workpiece.

Because the sheet-metal shop is configured as a flow shop with two machines, a fifth starting solution can be calculated by a procedure inspired by the method of Johnson [14] for a pure flow shop. First, the different sheets are composed as described above by using the workpiece order as given in the problem data input. Then, the cutting time and bending time for each sheet is calculated (without any set-up time). Finally, the method of Johnson is applied: sheets with small cutting times are placed in the beginning of the sequence and sheets with a small bending time are placed at the end of the sequence.

Another method of grouping together the workpieces of the same material and thickness is analogous to the longest processing time rule for minimising the makespan on a number of identical parallel machines. First, the minimum required number of sheets $(\kappa)$ is calculated by dividing the total area of the parts by the usable area of a sheet and rounding it up. When this number is equal to one, all workpieces of that material and thickness can be assigned to a single sheet. Otherwise, jobs of identical workpieces are ordered by decreasing workpiece area, they are consecutively assigned to the sheet with the least used area so far. When not all workpieces of a job can be assigned to a single sheet, the following sheet with the least used area so far is selected for further assignment. It is possible that not all workpieces of all jobs can be assigned to $\kappa$ sheets. In that case, an additional sheet is used for the rest of the workpieces. Because during this construction, no set-up or processing time parameters are taken into account, the resulting set of sheets is reordered based on the method of Johnson, described above.

So, the multi-start version will start from the six different initial seeds, as defined above. For each initial solution that is constructed, a combination of the five neighbourhoods is used. An overview of the solution method is presented in Algorithm 1.

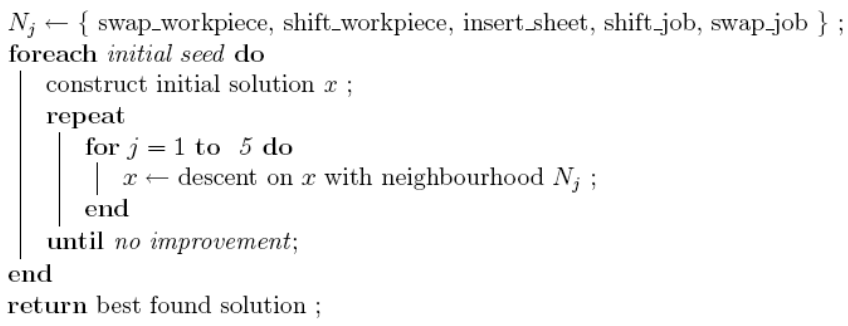

Algorithm 1. VNS for the single-machine problem.

\section{THE MULTIPLE-MACHINE CLASSES}

For the multiple-machine classes, a solution is specified by a permutation of the integers $1, \ldots, n$ with $n$ the number of workpieces. This permutation indicates the order in which the different workpieces will be handled. The workpieces are grouped together on a number of sheets. The solution also determines the laser machine and the press brake each sheet will be processed on. As in the two-stage approach, all workpieces of a sheet are cut by the same laser machine and are bent by the same press brake.

In the developed VNS procedure, eight different neighbourhoods structures are applied. Firstly, the shift_and swap_workpiece and the shift_- and swap_job moves are carried out. These four structures are identical to the ones defined for the single-machine class. But when a workpiece is moved from one sheet to another, handled by a different laser machine and/or a different press brake, the assigned laser machine and/or press brake is changed accordingly.

Instead of the rather complex insert_sheet neighbourhood for the single-machine class, a simpler shift_sheet neighbourhood is used. In this neighbourhood, just a single sheet is removed from one position in the sequence and inserted at another position (either before or after the original position). In addition, the swap_sheet neighbourhood is defined, swapping two sheets which are not required to be adjacent.

With these six moves, the sequence of the different workpieces and sheets are changed, but the assigned laser machines or press brakes to sheets are not. Therefore, two additional moves are defined. During the first move (shift_brake) the press brake that bends the workpieces from the last sheet of the production plan (and thus determines the makespan value) is considered. The sheets assigned to this critical press brake are taken out of the sequence one by one, and inserted into the sequence of another non-critical press brake. All positions in the sequences of the other press brakes are considered until an improvement is found. Then, the move is carried out and the search continues with the next sheet, or the search restarts if the critical press brake is changed. If for none of the positions an improvement can be found, the sheet is swapped with a sheet on a non-critical press brake: the sheet is inserted at the same position on a non-critical press brake and from this press brake a sheet is 
moved to the critical press brake. Again, once an improvement is detected, the move is carried out and the search continues as in the first case. An analogous procedure ( shift_laser) is defined for moving sheets between different laser machines.

Another issue is the initialisation of the search with a first solution. For such a solution, the following elements have to be determined: the composition of the sheets, the sequence of the workpieces, the assigment of the sheets to the different laser machines and the different press brakes. For the composition of the sheets and the sequence of the workpieces, the same procedures as for the single-machine class are used. This gives six different starting solutions. For the assignment to the different press brakes a simple round robin is used. A similar method is used for the assignment to the different laser machines.

In an alternative method, the assigment of the sheets to the different press brakes is based on the longest processing time rule (Baker and Trietsch [15]). For each sheet, the total bending time is calculated and the sheets are ordered by decreasing total bending time. They are then consecutively assigned to the first available press brake. For the multiple laser machine class of problems, the sheets are divided between the different laser machines based on the type of material: the sheets of the material requiring the largest total cutting time are assigned to the fast laser machine(s), the other sheets to the slower laser machine(s). By this division, the additional set-up time on a laser machine when switching materials is avoided. This assignment is not used when the problem instance contains sheets of only one type of material.

In the multi-start solution procedure (Algorithm 2) with twelve different starting solutions, a combination of all neighbourhoods is used. For each initial solution constructed, the following neighbourhoods are searched in the indicated order: swap_workpiece, shift_workpiece, swap_job, shift_job, swap_sheet, shift_sheet and shift_brake. For the multiple laser machine class, the shift_laser neighbourhood is also searched. When during one of these descent procedures an improvement is determined, the complete cycle with the seven (eight) neighbourhoods is repeated until no improvement can be found with one of the neighbourhoods.

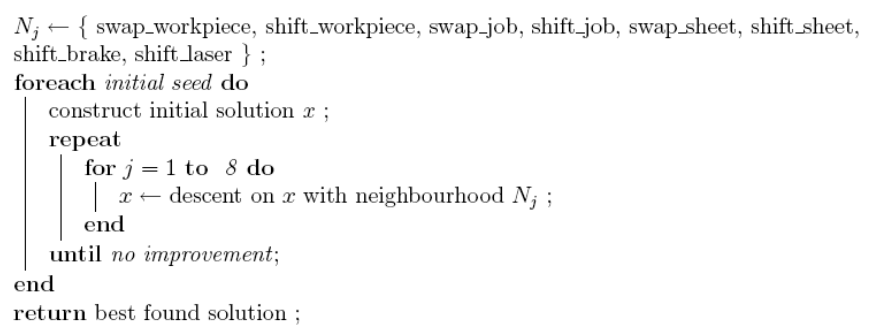

Algorithm 2. VNS for the multiple-machine problem.

\section{BICRITERIA SCHEDULING}

Usually, the construction of a production plan that scores well on both criteria, makespan and flow time, is necessary. This can be achieved by using a bicriteria approach. In such a problem setting there does not exist in general a unique optimal solution but a set of efficient solutions (also called the Pareto curve). A schedule $S$ is said to be efficient with respect to $\sum F_{j}$ and $C_{\max }$ if there does not exist any schedule $S^{\prime} \quad$ such that $\quad \sum F_{j}\left(S^{\prime}\right) \leq \sum F_{j}(S) \quad$ and $C_{\max }\left(S^{\prime}\right) \leq C_{\max }(S)$. Because the calculation of the Pareto curve requires large computational efforts, an approximation can be obtained by the budget approach: one criterion is minimised while the other does not exceed a budget. By iteratively incrementing the budget a number of solutions can be calculated resulting in an approximation of the Pareto curve. During each iteration $(k=1, \ldots, 5)$ the total flow time is minimised by the VNS procedure described in the previous sections:

\section{minimise $\sum F_{j}^{(k)}$}

with the additional constraint $\quad C_{\max }^{(k)} \leq C_{\max }^{(0)}+k \frac{C_{\max }^{(6)}-C_{\max }^{(0)}}{5}$

with $\left\{\begin{array}{l}C_{\max }^{(0)} \text { makespan by minimising makespan with a corresponding flow time } \sum F_{j}^{(0)} \\ C_{\max }^{(0)} \text { makespan by minimising flow time with a corresponding flow time } \sum F_{j}^{(6)} .\end{array}\right.$

When just one solution for the bicriteria problem is preferred, the two criteria can be combined. The most common combinations are the following. In the simultaneous approach, a single objective function is constructed by forming a weighted linear combination of the two relevant criteria: $\gamma C_{\max }+(1-\gamma) \frac{\sum F_{j}}{|S|}$ with $|S|$ the number of sheets and $\gamma$ a weight between 0.0 and 1.0. Mean flow time is considered in this linear combination because, in that way, the two terms are of the same magnitude. In the hierarchical approach, criteria are ranked in order of importance and then consecutively optimised. For the problem considered, it is desirable to minimise the makespan as the primary and minimise total flow time as the secondary objective. Thus, a production plan is constructed for which the total flow time is minimised, subject to the constraint that no deterioration of the makespan is allowed. Less interesting from a practical point of view is finding a schedule for which the makespan is minimised, subject to the constraint that no reduction in the total flow time is possible. For companies in the sheetmetal industry, the minimised makespan is often more important than the minimised total flow time.

In the simultaneous approach, the VNS method, described in the previous sections, is applied where the moves are evaluated according to the linear combination objective function. In the hierarchical approach, the VNS method is first used to optimise makespan. Then the VNS method is applied to the resulting solution to optimise the total flow time subject to the additional constraint that makespan does not get worse.

In Fig. (3) the solutions of the different approaches are shown for a single-machine problem instance consisting of 17 workpieces each with a thickness of $1 \mathrm{~mm}$ resulting in a schedule with 6 sheets. Instead of the solution values the percentage deviations are shown. For the hierarchical 
percentage deviations:

$$
\begin{aligned}
& P D M S=100 \frac{C_{\max }^{(k)}-C_{\max }^{(0)}}{C_{\max }^{(0)}} \\
& P D F T=100 \frac{\sum F_{j}^{(k)}-\sum F_{j}^{(6)}}{\sum F_{j}^{(6)}}
\end{aligned}
$$

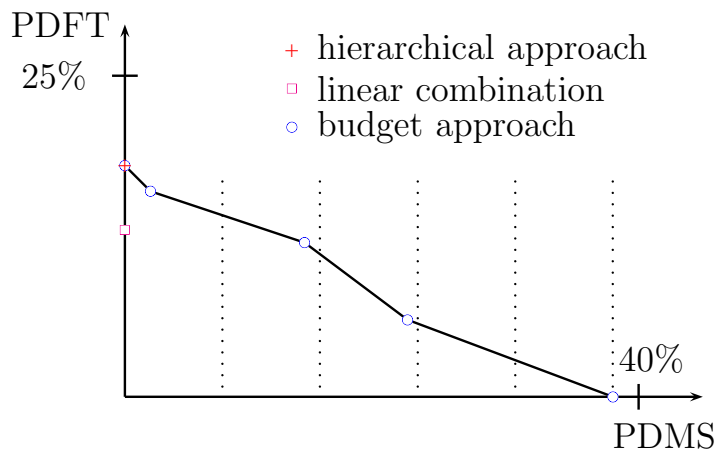

Fig. (3). Several solution approaches for the bicriteria optimisation problem.

approach no deviation is present for the makespan value because makespan minimisation is the primary criterion. For the secondary criterion, quite a large deviation is observed. With the linear combination approach, a schedule with a makespan equal to the best known is obtained and the total flow time is smaller compared to the one resulting from the hierarchical approach. Also shown in Fig. (3) are five different points of the budget approach. The first point is equal to schedule obtained with the hierarchical approach, because for this first point no budget is allowed on the makespan value. For the following points, better flow time values correspond to worse makespan values.

\section{COMPUTATIONAL EXPERIENCE}

For the computational tests, the VNS technique was coded in C and run on a HP 9000/rx3600 computer. A number of cases has been worked out to develop the VNS procedure for integrated production planning. The different test cases have been extensively used to evaluate the effectiveness of different alternatives. The workpieces included in these cases can be categorised as either complex parts $(C)$ or standard profiles $(P)$. Two materials, i.e. steel $(S)$ and stainless steel (SS) and five thicknesses ranging from $1 \mathrm{~mm}$ to $6 \mathrm{~mm}$ are used. In reality, some companies focus on piecewise production of complex parts, while others mainly produce larger series. Different combinations of parts have been included in the test set. The kind of workpiece and the used materials are indicated in Table $\mathbf{2}$ for each test case. Batch sizes range from one single part to dozens of parts, including different materials and sheet thicknesses. In total, ten different instances are used for the development of the procedure. In addition, five instances, each consisting of one hundred workpieces, are constructed for verifying the final procedure (Tables 9 and 10). The test cases "mixed1", "mixed2" and "small" contain both complex parts and standard profiles with thicknesses equal to $1 \mathrm{~mm}$, $1.5 \mathrm{~mm}$ and $2 \mathrm{~mm}$. Parts with thicknesses $3 \mathrm{~mm}$ and $6 \mathrm{~mm}$ are included in the test case "large" and the test case "profile" consists of a number of standard profiles. In these five problem instances, workpieces of steel and stainless steel are included.

For all instances, the VNS procedure is executed a number of times, firstly for minimising the makespan with as secondary criterion the total set-up time, and secondly for minimising the total flow time at the press brake(s). In both cases, the makespan, the total set-up time and the total flow time of the resulting production plan are calculated. In addition, the VNS procedure is executed for the bicriteria optimisation approaches: firstly for the linear combination approach with $\gamma=0.5$ and secondly for the hierarchical approach.

Table 2 presents the results for the single laser machine/single press brake class (SLSP) for the single criterion problems. The column labeled with $|S|$ gives the number of required sheets, determined by the new method. The table shows the percentage deviation of the makespan (PDMS), of the total set-up time (PDST) and of the total flow time on the press brake (PDFT) resulting from the first run of VNS (indicated by superscript ${ }^{(v)}$ in the following formulas) when compared with the makespan, the total setup time and the total flow time resulting from solving the mathematical programming model of Verlinden et al. [2] (indicated by superscript ${ }^{(m)}$ ). Note that in this mathematical programming model the objective is minimising the total setup time.

$$
\begin{aligned}
& P D M S=100 \frac{C_{\max }^{(v)}-C_{\max }^{(m)}}{C_{\max }^{(m)}} \\
& P D S T=100 \frac{\left(\sum s_{f g}\right)^{(v)}-\left(\sum s_{f g}\right)^{(m)}}{\left(\sum\left(s_{f g}\right)^{(m)}\right.} \\
& P D F T=100 \frac{\left(\sum F_{k}\right)^{(v)}-\left(\sum F_{k}\right)^{(m)}}{\left(\sum\left(F_{k}\right)^{(m)}\right.}
\end{aligned}
$$

Table 2. Results of SLSP

\begin{tabular}{|c|c|c|c|c|c|c|c|}
\hline & $\boldsymbol{n}$ & $\boldsymbol{I} \boldsymbol{S} \mathbf{I}$ & Material & Kind & PDMS & PDST & PDFT \\
\hline \hline thick_1mm & 17 & 6 & $\mathrm{~S}+\mathrm{SS}$ & $\mathrm{C}+\mathrm{P}$ & 0.00 & 0.00 & -3.51 \\
\hline thick_1.5mm & 17 & 3 & $\mathrm{~S}+\mathrm{SS}$ & $\mathrm{C}+\mathrm{P}$ & -3.53 & 0.00 & -1.64 \\
\hline thick_2mm & 12 & 2 & $\mathrm{~S}+\mathrm{SS}$ & $\mathrm{C}+\mathrm{P}$ & 0.00 & 0.00 & -4.67 \\
\hline thick_3mm & 80 & 3 & $\mathrm{SS}$ & $\mathrm{C}+\mathrm{P}$ & -12.01 & 29.05 & -8.58 \\
\hline thick_6mm & 150 & 18 & $\mathrm{SS}$ & $\mathrm{C}+\mathrm{P}$ & -0.45 & 0.00 & -1.50 \\
\hline thick_small & 46 & 11 & $\mathrm{~S}+\mathrm{SS}$ & $\mathrm{C}+\mathrm{P}$ & -12.44 & 17.26 & -1.15 \\
\hline small_profiles & 24 & 8 & $\mathrm{SS}$ & $\mathrm{P}$ & -0.84 & 5.58 & -4.45 \\
\hline small_complex & 22 & 7 & $\mathrm{~S}+\mathrm{SS}$ & $\mathrm{C}$ & -6.25 & 10.45 & -16.37 \\
\hline large_profiles & 152 & 15 & $\mathrm{SS}$ & $\mathrm{P}$ & -0.15 & 0.00 & 9.84 \\
\hline large_complex & 78 & 8 & $\mathrm{SS}$ & $\mathrm{C}$ & 0.00 & 0.00 & -0.34 \\
\hline average & & & & & -3.57 & 6.23 & -3.25 \\
\hline
\end{tabular}


It is obvious that the new procedure improves on the makespan value (seven instances) or finds the same value, because the main objective is now minimising the makespan while previously the total set-up time was minimised. For some instances, the improvement of the makespan comes with a deterioration of the total set-up time, and this deterioration can be quite large (almost thirty percent for the "thick_3mm" case). The required computation time is less than a second for the smaller instances (with less than fifty workpieces). The largest required computation time is less than 10 seconds.

For the "thick_6mm" instance, a solution is constructed that only requires 18 sheets instead of 19 (as is the case with the original method), by starting from the initial sequence where the sheets are composed based on the largest workpiece area. As already indicated, a minimal number of used sheets is very important in the sheet-metal shop. In this case, there is no negative effect on the value of the makespan.

Although the first run of VNS minimises the makespan, the resulting sequence also shows an improvement on the total flow time at the press brake when compared with the corresponding value of the mathematical programming model. On average, an improvement of $3.25 \%$ is obtained and only for one instance, a worse total flow time is observed.

Table 3 compares the results of the single criterion optimisation with the linear combination and hierarchical approach for the bicriteria optimisation. The percentage deviations on makespan (MS_x) and on total flow time (FT_x) are shown by comparing the results of the first run of VNS (minimising makespan, indicated by ${ }^{(0)}$ ) and a second run of VNS, indicated by ${ }^{(x)}$, either minimising total flow time (with ' $\mathrm{x}$ ' equal to ' $\mathrm{S}$ '), or the linear combination of makespan and mean flow time (with ' $x$ ' equal to ' $L$ '), or the hierarchical objective function (with ' $\mathrm{x}$ ' equal to ' $\mathrm{H}$ '):

$$
\begin{aligned}
& M S \_x=100 \frac{C_{\max }^{(x)}-C_{\max }^{(0)}}{C_{\max }^{(0)}} \\
& F T \_x=100 \frac{\left(\sum F_{k}\right)^{(x)}-\left(\sum F_{k}\right)^{(0)}}{\left(\sum\left(F_{k}\right)^{(0)}\right.} \text { with } x \in\{S, L, H\}
\end{aligned}
$$

Table 3. Results of SLSP: Bicriteria Approaches

\begin{tabular}{|c|c|c|c|c|c|c|}
\hline & \multicolumn{2}{|c|}{ Single Criterion } & \multicolumn{2}{c|}{ Linear Combination } & \multicolumn{2}{c|}{ Hierarchical } \\
\cline { 2 - 7 } & MS_S & FT_S & MS_L & FT_L & MS_H & FT_H \\
\hline \hline thick_1mm & 38.35 & -15.44 & 0.00 & -4.08 & 0.00 & 0.00 \\
\hline thick_1.5mm & 11.41 & -12.59 & 0.00 & -2.90 & 0.00 & -1.41 \\
\hline thick_2mm & 0.00 & 0.00 & 0.00 & 0.00 & 0.00 & 0.00 \\
\hline thick_3mm & 25.51 & -8.89 & 1.10 & -1.51 & 0.00 & 0.00 \\
\hline thick_6mm & 0.15 & -6.56 & 0.08 & -6.61 & 0.00 & -4.87 \\
\hline thick_small & 9.35 & -14.64 & 0.00 & -0.62 & 0.00 & -0.30 \\
\hline small_profiles & 11.32 & -19.10 & 1.34 & -17.74 & 0.00 & -5.55 \\
\hline small_complex & 8.98 & -18.36 & 5.29 & -12.71 & 0.00 & -1.90 \\
\hline large_profiles & 0.50 & -16.11 & 0.50 & -16.47 & 0.00 & 0.00 \\
\hline large_complex & 0.00 & -2.78 & 0.00 & -2.78 & 0.00 & -2.78 \\
\hline average & 10.56 & -11.45 & 0.83 & -6.54 & 0.00 & -1.68 \\
\hline
\end{tabular}

When the objective function is minimising the total flow time, a large improvement of this value can be observed, the average is $11.45 \%$. As can be expected, this improvement comes with a deterioration on the value for makespan, on average equal to $10.56 \%$. When a bicriteria optimisation approach is taken, the results with the linear combination of makespan and mean flow time show that quite a large improvement can be obtained for the total flow time with only a small deterioration on makespan. The hierarchical approach, with makespan as primary criterion, still results in a small improvement for the total flow time, on average $1.68 \%$.

Table 4 presents the results for the multiple press brake classes, both related machines (SLRP) and unrelated machines (SLUP). Note that in the mathematical programming models for the multiple-machine classes the objective is minimising makespan. The column labeled with $|B|$ gives the number of available press brakes. There is only a small difference between the class of related parallel press brakes and the class with the unrelated press brakes.

While comparing with the two-stage approach of the mathematical programming model, a small improvement in makespan is observed for only a few instances. Quite large improvements are found for the sum of set-up times. The main reason is that neutral moves for the makespan value, but with a smaller total set-up time, are not accepted in the two-stage approach. In the two-stage approach the composition of the sheets is fixed by the first stage whereby the sum of set-up times on a single machine is minimised. In the second stage the sheets are assigned to the available press brakes and this can result in larger change-over times between sheets on a press brake. In the integrated approach, these change-over times can decrease by changing the sequence of the workpieces on the individual sheets.

Most instances can be solved in less than ten seconds. Only the large instances ("thick_3mm" and "thick_6mm") require more computation time (respectively 24 and 11 seconds). Calculating a short term production plan for one working day in less than a few minutes is acceptable in an industrial environment.

Tables 5 and 6 show the results of the single criterion optimisation and bicriteria optimisation for the multiple press brake classes. As for the single-machine class, an improvement in total flow time is observed (column FT_S) for the single criterion optimisation (optimising total flow time). But for most instances, this comes with a deterioration on makespan (column MS_S). These deviations are again quite large, in most cases more than $10 \%$. In order to reduce the deterioration on makespan, a bicriteria optimisation approach can be taken. The results from the linear combination of makespan and mean flow time show that still quite a large improvement can be obtained for the total flow time, i.e. more than $7 \%$. When no deterioration on makespan is allowed at all, the hierarchical approach can get a small improvement for total flow time: on average $4 \%$ (SLRP) and $5 \%$ (SLUP). 
Table 4. Results of Single Laser Machine/Multiple Press Brakes

\begin{tabular}{|c|c|c|c|c|c|c|c|c|}
\hline \multicolumn{2}{|c|}{} & \multicolumn{3}{c|}{ Related Press Brakes } & \multicolumn{3}{c|}{ Unrelated Press Brakes } \\
\hline & $|\boldsymbol{I}|$ & $|\boldsymbol{B}|$ & PDMS & PDST & PDFT & \multicolumn{2}{c|}{ PDMS PDST } & \multicolumn{2}{c|}{ PDFT } \\
\hline \hline thick_1mm & 6 & 2 & 0.00 & -12.77 & -4.09 & 0.00 & -12.77 & -11.98 \\
\hline thick_1.5mm & 3 & 2 & 0.00 & 0.00 & 0.00 & 0.00 & 0.00 & 0.00 \\
\hline thick_2mm & 2 & 2 & 0.00 & -30.34 & 0.00 & 0.00 & 0.00 & 0.00 \\
\hline thick_3mm & 3 & 2 & -11.89 & -19.09 & 11.74 & -11.73 & -10.82 & 11.19 \\
\hline thick_6mm & 18 & 5 & -0.42 & 0.00 & -5.26 & -0.42 & 0.00 & -5.31 \\
\hline thick_small & 11 & 4 & 0.00 & -37.18 & -2.96 & 0.00 & -34.05 & -5.06 \\
\hline small_profiles & 8 & 3 & 0.00 & -39.38 & 4.01 & 0.00 & -39.38 & 6.70 \\
\hline small_complex & 7 & 3 & 0.00 & -36.03 & 2.15 & -1.10 & -42.42 & -7.36 \\
\hline large_profiles & 15 & 4 & -0.15 & -22.49 & 4.20 & -0.15 & -22.49 & 4.23 \\
\hline large_complex & 8 & 3 & 0.00 & -43.62 & -1.25 & 0.00 & -18.98 & 0.07 \\
\hline average & & & -1.25 & -24.09 & 0.85 & -1.34 & -18.09 & -0.75 \\
\hline
\end{tabular}

Table 5. Results of SLRP: Bicriteria Approaches

\begin{tabular}{|c|c|c|c|c|c|c|}
\hline & \multicolumn{2}{|c|}{ Single Criterion } & \multicolumn{2}{c|}{ Linear Combination } & \multicolumn{2}{c|}{ Hierarchical } \\
\cline { 2 - 7 } & MS_S & FT_S & MS_L & FT_L & MS_H & FT_H \\
\hline \hline thick_1mm & 38.35 & -7.13 & 0.45 & -3.51 & 0.00 & -0.73 \\
\hline thick_1.5mm & 6.75 & -2.17 & 0.00 & 0.00 & 0.00 & 0.00 \\
\hline thick_2mm & 0.00 & 0.00 & 0.00 & 0.00 & 0.00 & 0.00 \\
\hline thick_3mm & 39.36 & -16.12 & 8.90 & -10.32 & 0.00 & 0.00 \\
\hline thick_6mm & 0.15 & -7.02 & 0.15 & -7.02 & 0.00 & -5.95 \\
\hline thick_small & 10.89 & -13.93 & 0.00 & -6.15 & 0.00 & -5.66 \\
\hline small_profiles & 11.32 & -19.98 & 0.00 & -18.70 & 0.00 & -18.70 \\
\hline small_complex & 21.76 & -14.77 & 0.00 & -7.99 & 0.00 & -4.38 \\
\hline large_profiles & 0.50 & -16.53 & 0.50 & -16.53 & 0.00 & -7.04 \\
\hline large_complex & 0.00 & -1.05 & 0.00 & -1.05 & 0.00 & -0.80 \\
\hline average & 12.91 & -9.87 & 1.00 & -7.13 & 0.00 & -4.33 \\
\hline
\end{tabular}

In Table 7, the results of the last class with multiple unrelated laser machines and multiple unrelated press brakes (ULUP) are shown. The column labeled with $|L|$ gives the number of available laser machines. An improvement can be observed in makespan (column PDMS) for a number of instances with the first run, while comparing values obtained with the two-stage approach. For a lot of these instances the decrease in the sum of set-up times is even larger (column PDST). Yet, there are also two instances where the solution is worse than with the two-stage approach. It is due to an extra sheet in the two-stage approach for the "thick_6mm" instance, This extra sheet only contains one workpiece and thus, it requires only a small bending time. By putting this sheet in the last position of the sequence, a smaller makespan is obtained. For the other instance ("small_profiles") the difference is smaller, but no specific explanation is found. As for the previous classes, the required computation time is quite modest, with a maximum of twenty seven seconds for the instance "thick_6mm".

Table 6. Results of SLUP: Bicriteria Approaches

\begin{tabular}{|c|c|c|c|c|c|c|}
\hline & \multicolumn{2}{|c|}{ Single Criterion } & \multicolumn{2}{c|}{ Linear Combination } & \multicolumn{2}{c|}{ Hierarchical } \\
\cline { 2 - 7 } & MS_S & FT_S & MS_L & FT_L & MS_H & FT_H \\
\hline \hline thick_1mm & 38.35 & -10.87 & 0.00 & -5.96 & 0.00 & -4.30 \\
\hline thick_1.5mm & 12.88 & -2.55 & 0.00 & 0.00 & 0.00 & 0.00 \\
\hline thick_2mm & 0.00 & 0.00 & 0.00 & 0.00 & 0.00 & 0.00 \\
\hline thick_3mm & 39.12 & -17.43 & 1.24 & -1.60 & 0.00 & -0.05 \\
\hline thick_6mm & 0.15 & -7.04 & 0.15 & -6.96 & 0.00 & -5.89 \\
\hline thick_small & 10.89 & -19.82 & 0.00 & -12.53 & 0.00 & -11.24 \\
\hline small_profiles & 11.32 & -22.67 & 0.00 & -21.17 & 0.00 & -20.17 \\
\hline small_complex & 21.76 & -12.44 & 0.00 & -4.44 & 0.00 & -0.03 \\
\hline large_profiles & 0.50 & -16.54 & 0.50 & -16.54 & 0.00 & -7.05 \\
\hline large_complex & 0.00 & -3.02 & 0.00 & -2.52 & 0.00 & -2.00 \\
\hline average & 13.50 & -11.24 & 0.19 & -7.17 & 0.00 & -5.07 \\
\hline
\end{tabular}

Table 8 compares the results of the single criterion optimisation with the bicriteria optimisations. As for the previous classes, the run of VNS with the single criterion minimising total flow time, gives a far better value for this total flow time, (on average $13.77 \%$ ) but at the expense of the makespan value (on average 18.93\%). With bicriteria optimisation, total flow time can be improved without a large deterioration of makespan. By using the linear combination objective function, an improvement of more than $8 \%$ on average can be observed for total flow time whereas the hierarchical approach results in an improvement of on average $3.79 \%$.

Tables 9 and 10 present the results for the additional instances. As in the previous tables, $|S|$ presents the number 
of sheets, $|L|$ the number of available laser machines (class ULUP) and $|B|$ the number of available press brakes (classes SLRP, SLUP and ULUP). Because the set "large" contains workpieces of $3 \mathrm{~mm}$ and $6 \mathrm{~mm}$ thickness, the total cutting time is quite large compared to the total bending time. Therefore, four laser machines are provided in the ULUP class.

Table 7. Results of Unrelated Laser Machines/Unrelated Press Brakes

\begin{tabular}{|c|c|c|c|c|c|c|c|}
\hline & $\boldsymbol{n}$ & $|\boldsymbol{S}|$ & $|\boldsymbol{L}|$ & $|\boldsymbol{B}|$ & PDMS & PDST & PDFT \\
\hline \hline thick_1mm & 17 & 6 & 2 & 2 & -14.13 & -25.55 & -1.86 \\
\hline thick_1.5mm & 17 & 3 & 1 & 2 & 0.00 & 0.00 & 0.00 \\
\hline thick_2mm & 12 & 2 & 1 & 2 & 0.00 & 0.00 & 0.00 \\
\hline thick_3mm & 80 & 3 & 1 & 2 & -11.73 & -10.82 & 11.89 \\
\hline thick_6mm & 150 & 18 & 3 & 5 & 0.77 & 0.00 & -0.65 \\
\hline thick_small & 46 & 11 & 2 & 4 & -4.69 & -16.64 & -3.99 \\
\hline small_profiles & 24 & 8 & 2 & 3 & 0.15 & 0.37 & -13.04 \\
\hline small_complex & 22 & 7 & 2 & 3 & -8.74 & -1.94 & 0.50 \\
\hline large_profiles & 152 & 15 & 3 & 4 & -0.18 & 0.00 & -5.49 \\
\hline large_complex & 78 & 8 & 2 & 3 & -3.28 & -15.81 & 11.40 \\
\hline average & & & & & -4.18 & -7.04 & -0.19 \\
\hline
\end{tabular}

Table 8. Results of ULUP: Bicriteria Approaches

\begin{tabular}{|c|c|c|c|c|c|c|}
\hline & \multicolumn{2}{|c|}{ Single Criterion } & \multicolumn{2}{c|}{ Linear Combination } & \multicolumn{2}{c|}{ Hierarchical } \\
\cline { 2 - 7 } & MS_S & FT_S & MS_L & FT_L & MS_H & FT_H \\
\hline \hline thick_1mm & 63.80 & -14.75 & 0.79 & -5.27 & 0.00 & -0.34 \\
\hline thick_1.5mm & 12.88 & -2.55 & 0.00 & 0.00 & 0.00 & 0.00 \\
\hline thick_2mm & 0.00 & 0.00 & 0.00 & 0.00 & 0.00 & 0.00 \\
\hline thick_3mm & 39.12 & -17.43 & 1.24 & -1.60 & 0.00 & -0.05 \\
\hline thick_6mm & 3.73 & -14.81 & 1.19 & -14.15 & 0.00 & -12.14 \\
\hline thick_small & 23.26 & -24.75 & 1.56 & -11.82 & 0.00 & -5.66 \\
\hline small_profiles & 18.68 & -12.36 & 0.57 & -6.00 & 0.00 & -1.84 \\
\hline small_complex & 22.93 & -15.90 & 4.48 & -8.71 & 0.00 & 0.00 \\
\hline large_profiles & 1.93 & -18.26 & 0.80 & -17.98 & 0.00 & -4.51 \\
\hline large_complex & 3.00 & -16.88 & 0.00 & -15.60 & 0.00 & -13.40 \\
\hline average & 18.93 & -13.77 & 1.06 & -8.11 & 0.00 & -3.79 \\
\hline
\end{tabular}

These tables confirm the previous findings. For the single laser machine/single press brake class and the multiple unrelated laser machines/unrelated press brakes quite large improvements in makespan are found by using the new VNS procedure (Table 9). For the single-machine class, this is due to the fact that our method searches for a minimum makespan value whereas the original method minimises the total set-up time at the press brakes. The integrated approach of the method for the multi-machine classes explains the better performance for these classes. Although, the improvements for the single laser machine/multiple press brakes are smaller.

There is one instance (mixed1) with opposite results. The main reason is that, with the new procedure, a solution is generated that requires 15 sheets where the original method constructs a plan with 16 sheets. A plan with a worse makespan value requiring less sheets is in most situations acceptable whenever the difference for this makespan is not that large. Also for the instances "small" and "profile" the original method constructs plans with more sheets, respectively 17 and 26 sheets. For these instances, the new method constructs a plan with less sheets resulting in a smaller makespan value. For the single-machine class and the multiple laser machines/multiple press brakes class, the improvement is quite large (in most cases larger than 5\%).

Table 9. Results for Additional Problem Instances: Minimising $C_{\max }$

\begin{tabular}{|c|c|c|c|c|c|c|c|c|}
\hline & $n$ & $|\boldsymbol{S}|$ & $|\boldsymbol{L}|$ & $\boldsymbol{I} \boldsymbol{B} \mid$ & SLSP & SLRP & SLUP & ULUP \\
\hline \hline mixed1 & 100 & 15 & 2 & 2 & 1.49 & -1.78 & 0.59 & 4.99 \\
\hline mixed2 & 100 & 15 & 2 & 2 & -7.11 & -0.23 & -0.23 & -5.20 \\
\hline small & 100 & 15 & 2 & 2 & -5.28 & -2.96 & -2.96 & -2.14 \\
\hline large & 100 & 10 & 4 & 2 & -0.16 & -0.16 & -0.16 & -1.77 \\
\hline profile & 100 & 23 & 2 & 2 & -7.53 & -2.24 & -2.24 & -5.34 \\
\hline average & & & & & -3.72 & -1.47 & -1.00 & -1.89 \\
\hline
\end{tabular}

Table 10 shows the average improvement on total flow time and average deterioration on makespan for the different classes. Quite large improvements can be obtained for the total flow time value when this performance measure is minimised instead of makespan (column FT_S). For three of the four classes, an average improvement of more than $30 \%$ is observed, whereas the makespan value deteriorates in most cases with less than $20 \%$ (column MS_S). The computational requirements for this second run are modest (less than thirty seconds) and in most cases, only a few seconds more are used compared to the computational requierements of the first run (minimising makespan). For each class and for each type of neighbourhood, more moves are accepted when the procedure is used for the minimisation of the total flow time. Especially, the shift_workpiece and the swap_sheet neigbourhoods result in a lot more improving moves. The largest difference in required CPU time is observed for the SLRP and SLUP classes. For these instances, almost twice the time is needed by the VNS procedure when minimising the total flow time. In these runs, the shift_brake move is carried out more frequently than in the runs for minimising the makespan. For the ULUP class, the number of times the shift_brake is improving, is comparable for both runs.

Finally, the bicriteria approaches indicate that with the linear combination objective function a large improvement for total flow time (FT_L) can be obtained with only a small deterioration on makespan (column MS_L). This 
improvement is not that large when the hierarchical approach is used, on average varying between $1.84 \%$ and $11.63 \%$.

Table 10. Bicriteria Approaches for Additional Problem Instances

\begin{tabular}{|c|c|c|c|c|c|c|}
\hline \multirow{2}{*}{} & \multicolumn{2}{|c|}{ SingleCriterion } & \multicolumn{2}{c|}{ LinearCombination } & \multicolumn{2}{c|}{ Hierarchical } \\
\cline { 2 - 7 } & MS_S & FT_S & MS_L & FT_L & MS_H & FT_H \\
\hline \hline SLSP & 12.00 & -30.56 & 1.77 & -25.99 & 0.00 & -1.84 \\
\hline SLRP & 17.95 & -28.06 & 2.16 & -23.12 & 0.00 & -9.19 \\
\hline SLUP & 20.73 & -31.08 & 2.88 & -26.39 & 0.00 & -11.63 \\
\hline ULUP & 15.21 & -33.61 & 1.97 & -28.03 & 0.00 & -4.46 \\
\hline
\end{tabular}

\section{CONCLUDING REMARKS}

This paper presents extensions and adaptations to the variable neighbourhood search methods for integrated production planning in a sheet-metal shop developed by Verlinden et al. [2]. The main objective function is makespan minimisation, but special care is taken for generating production plans with a minimal number of sheets because waste material minimisation is of utmost importance in the sheet-metal shop. For many problem instances a better production plan is generated and, in some cases, plans requiring less sheets are generated. The required computation time for generating a production plan corresponding to a day's work, is less than a minute and thus acceptable in practice.

In addition, the developed procedure is used to minimise the total flow time. It is obvious that for almost every instance a plan with a far better total flow time is generated, but with a worse makespan value. The assumption frequently made in industry and text books that a production plan with a good makespan also scores quite well against other performance criteria related to completion times (e.g. total flow time) appears to be questionable.

Using bicriteria optimisation is a solution to that problem. The presented heuristic method can generate a production plan that minimises the makespan with a secondary criterion, i.e. the total flow time. The results show that a reduction in total flow time is possible without deteriorating makespan. When a small deterioration of makespan is acceptable, quite a large improvement on total flow time can be realised by optimising a linear combination of makespan and mean flow time.

A future research topic is to optimise the laser cutting time. By considering the directions in which cutting lines are done, several non-active movements of the laser between cutting lines can be eliminated. Another related optimisation deals with sharing the same cutting line by two adjacent workpieces. This also reduces the cutting time and can have a positive effect on the number of required sheets.

On a more methodological level, constructing a selfadapting heuristic method could be considered. In such a method the inclusion of certain neighbourhood types and the sequence in which these neighbourhoods are searched, depend on specific problem characteristics. For example, when the parts comprise mainly simple profiles of the same material, requiring the same press brake, fewer specific neighbourhoods might be sufficient. If however, different complex parts of different materials and sheet thickness and with different requirements for the type of press brake are included, more types of neighbourhoods will most likely be needed to generate good solutions.

\section{APPENDIX}

Model for minimising the makespan $C_{\max }$.

By defining variables

$x_{k l m t}= \begin{cases}1 & \text { sheet } l \text { follows sheet } k \text { on machine } m \text { at stage } t \\ 0 & \text { otherwise, }\end{cases}$

$z_{i j k}=\left\{\begin{array}{l}1 \text { workpiece } j \text { follows workpiece } i \text { on sheet } k \\ 0 \quad \text { otherwise }\end{array}\right.$

$u_{\mathrm{i}}$ : integer counter to prevent subtours,

and using the following parameters

- $\quad h, i, j:$ workpiece indices,

- $\quad k, l, g:$ sheet indices,

- $\quad m$ : machine index,

- $t$ : stage index $(1=$ cutting stage, $2=$ air bending stage),

- $\quad c_{i}$ : cutting time of workpiece $i$,

- $\quad p_{i}$ : bending time of workpiece $i$,

- $\quad s_{i j}$ : sequence-dependent set-up time between workpiece $i$ and $j$

- $\quad A_{i}$ : surface of workpiece $i$,

- $\quad C_{k}$ : capacity of sheet $k$,

- $\quad \alpha$ : maximum occupation rate of a sheet,

- $\quad P_{k m t}$ : production time of sheet $k$ on machine $m$ at stage $t$,

- $\quad S_{k l t}$ : sequence-dependent set-up time between sheet $k$ and $l$ at stage $t$,

- $\quad T_{k m t}$ : starting time for sheet $k$ on machine $m$ at stage $t$,

- $Q, R:$ large integer values,

- $\quad I$ : all workpieces,

- $\quad K:$ all sheets,

- $\quad M_{t}$ : all machines at stage $t$, with $m_{A}$ indicating the laser machine and $m_{B}$ the press brake;

we obtain the following formulation: 


$$
\begin{aligned}
& \min C_{\max } \\
& \text { s.t. } \quad \sum_{l=0}^{|K|} \sum_{m=1}^{\left|M_{t}\right|} x_{k l m t}=1 \\
& \forall k \in K_{0} ; t \in\{1,2\} \\
& \sum_{l=1}^{|K|} x_{0 l m t}=1 \quad \forall m \in M_{t} ; t \in\{1,2\} \\
& \sum_{k=1}^{|K|} x_{k 0 m t}=1 \quad \forall m \in M_{t} ; t \in\{1,2\} \\
& \sum_{k=0}^{|K|} x_{k g m t}-\sum_{l=0}^{|K|} x_{g l m t}=0 \quad \forall g \in K_{0} ; \forall m \in M_{t} ; t \in\{1,2\} \\
& T_{l m t}-T_{k m t}+R\left(1-x_{k l m t}\right) \geq P_{k m t}+S_{k l t} \quad \forall k, l \in K_{0} ; \\
& \forall m \in M_{t} ; t \in\{1,2\} \\
& T_{k m_{B} 2}-T_{k m_{A}{ }^{1}}-P_{k 11} \geq 0 \quad \forall k \in K_{0} ; \\
& \forall m_{A} \in M_{1} ; \forall m_{B} \in M_{2} \\
& C_{\max }-T_{l m 2}-S_{k l 2} x_{k l m 2}-P_{j m 2} x_{k l m 2} \geq 0 \quad \forall k, l \in K_{0} ; \forall m \in M_{2} \\
& \sum_{j=0}^{|I I|} \sum_{k=1}^{|K|} z_{i j k}=1 \quad \forall i \in I_{0} \\
& \sum_{j=1}^{|l|} z_{0 j k}=1 \quad \forall k \in K \\
& \sum_{i=1}^{|l|} z_{i 0 k}=1 \quad \forall k \in K \\
& \sum_{i=0}^{|l|} z_{i h k}-\sum_{j=0}^{|l|} z_{h j k}=0 \quad \forall h \in I ; \forall k \in K \\
& \sum_{i=0}^{|I|} \sum_{j=1}^{|l|} A_{j} z_{i j k} \leq \alpha C_{k} \quad \forall k \in K \\
& \sum_{i=0}^{|l|} \sum_{j=1}^{|l|} c_{j} z_{i j k}=P_{k m_{A} 1} \quad \forall k \in K \\
& \sum_{i=0}^{\mid I I I} \sum_{j=1}^{\mid I I}\left(s_{i j}+p_{j}\right) z_{i j k}=P_{k m_{B} 2} \quad \forall k \in K \\
& u_{i}-u_{j}+Q z_{i j k}+\left(Q-A_{i}-A_{j}\right) z_{i j k} \leq Q-A_{j} \quad \forall i, j \in I ; i \neq j ; \forall k \in K \\
& A_{i} \leq u_{i} \leq Q \\
& \forall i \in I \\
& x_{k l p t} \in\{0,1\} \\
& \forall k, l \in K ; \forall m \in M_{t} ; t \in\{1,2\} \\
& z_{i j k} \in\{0,1\} \\
& \forall i, j \in I ; \forall k \in K
\end{aligned}
$$

The first constraint (2) expresses that each sheet needs to be processed at each stage. Constraints (3), (4) and (5) generate the production sequence of the sheets for each machine. Constraints (6) and (7) ensure that a sheet is only processed when its predecessor is finished. Constraint (8) calculates the makespan for the batch of sheets. Constraint (9) ensures that every workpiece is included on a sheet and hence produced. Constraints (10), (11) and (12) generate the production sequence of the workpieces for each sheet. Capacity constraint (13) assures that a sheet is filled to at most its available capacity. Constraints (14) and (15) calculate the total cutting time and the total bending time respectively, for each sheet. Subtour elimination constraints (16) and (17) make sure that no subtours are created for a sheet. Constraints (18) and (19) limit the decision variables to binary values.

\section{REFERENCES}

[1] Verlinden B, Cattrysse D, Van Oudheusden D. Integrated sheetmetal production planning for laser cutting and bending. Int $\mathbf{J}$ Prod Res 2007; 45(2): 369-83.

[2] Verlinden B, Cattrysse D, Crauwels H, Van Oudheusden D. The development and application of an integrated production planning methodology for sheet metal working SMEs. Prod Plan Control 2009; 20(7): 649-63. 
[3] Toth P, Vigo D, Eds. The vehicle routing problem. Siam monographs on discrete mathematics and applications. Philidalphia, PA: SIAM 2002.

[4] Golden BL, Raghavan S, Wasil EA, Eds. The vehicle routing problem: latest advances and new challenges. Operations Research/Computer Science Interfaces Series, Berlin: Springer 2008.

[5] Mladenović N, Hansen P. Variable neighborhood search. Comput Oper Res 1997; 24(11): 1097-1100.

[6] Hansen P, Mladenović N. Variable neighborhood search: principles and applications. Eur J Oper Res 2001; 130(3): 449-67.

[7] T'kindt V, Billaut J-C. Multicriteria scheduling problems: a survey. RAIRO Oper Res 2001; 35: 143-63.

[8] Gupta JND, Ho JC. Minimizing flowtime subject to optimal makespan on two identical parallel machines. Pesq Oper 2000; 20(1): 5-18.
[9]

11] Hendizadeh SH, ElMekkawy TY, Wang GG. Bi-criteria scheduling of a flowshop manufacturing cell with sequence dependent setup times. Eur J Ind Eng 2007; 1(4): 391-413.

[12] Rayward-Smith VJ, Osman IH, Reeves CR, Smith GD. Modern heuristic search methods. Chichester: John Wiley \& Sons 1996.

[13] Hansen P, Mladenović N, Moreno-Pérez JA. Variable neighborhood search (Editorial of a special issue on VNS). Eur J Oper Res 2008; 191: 593-5.

[14] Johnson SM. Optimal two- and three-stage production schedules with setup times included. Nav Res Logist 1954; 1(1): 61-8.

[15] Baker KR, Trietsch D. Principles of sequencing and scheduling. New York: John Wiley \& Sons 2009.

(C) Crauwels et al.; Licensee Bentham Open.

This is an open access article licensed under the terms of the Creative Commons Attribution Non-Commercial License (http://creativecommons.org/licenses/by-nc/ 3.0/) which permits unrestricted, non-commercial use, distribution and reproduction in any medium, provided the work is properly cited. 